\title{
A política editorial da RevSALUS sobre as autorias: uma reflexão crítica
}

\author{
Ricardo Jorge Dinis-Oliveira ${ }^{1,2,3^{*} \text { iD }}$ \\ 'INFACTS - Instituto de Investigação e Formação Avançada em Ciências e Tecnologias da Saúde, Departamento de Ciências, Instituto Universitário de Ciências da Saúde (IUCS- \\ CESPU), Gandra, Portugal. \\ ²Departamento de Ciências da Saúde Pública e Forenses e Educação Médica, Faculdade de Medicina, Universidade do Porto, Porto, Portugal. \\ ${ }^{3}$ UCIBIO-REQUIMTE, Laboratório de Toxicologia, Departamento de Ciências Biológicas, Faculdade de Farmácia, Universidade do Porto, Porto, Portugal. \\ *Editor Chefe da RevSALUS * ${ }^{*}$ ricardo.dinis@iucs.cespu.pt
}

Recebido em: 12 março 2021; Revisto em: 25 março 2021; Aceite em: 26 março 2021

\section{Resumo}

Durante o ano de 2020 a RevSALUS continuou a trilhar o seu caminho de afirmação como revista científica para a Lusofonia. A RevSALUS recebeu através da sua plataforma eletrónica de gestão do processo editorial Open Journal System um total de 33 artigos, dos quais 17 foram publicados nos Volume 2: Números 1 e 2, lançados em junho e dezembro de 2020, respetivamente. Viu também reconhecido o seu projeto científico através da sua aceitação pela Publons Reviewer Recognition e passou a integrar no seu processo de revisão editorial dos artigos o serviço de iThenticate: plagiarism detection. Este primeiro editorial de 2021 da RevSALUS e dedicado a autoria de artigos científicos e como a RevSALUS se revê neste domínio, nomeadamente quanto ao cumprimento dos critérios definidos pelo International Commitee of Medical Journals Editors (ICMJE).

Palavras-chave: Autorias; Colaboradores; Guidelines do International Commitee of Medical Journals Editors (ICMJE); Critérios; Hyperauthorship.

A autoria dos trabalhos científicos, como são o caso dos artigos, confere crédito e tem importantes implicações académicas, sociais e financeiras. Mas a autoria implica também responsabilidade e responsabilização pelo trabalho publicado (International Committee of Medical Journal Editors - ICMJE, 2019).

Este primeiro editorial de 2021 da RevSALUS é dedicado à autoria de artigos científicos e como a RevSALUS se revê neste domínio. De acordo com o International Commitee of Medical Journals Editors (ICMJE), nas suas recomendações para elaboração, redação, edição e publicação de trabalhos académicos em revistas médicas (2015b; International Committee of Medical Journal Editors ICMJE, 2019), as quais subscrevemos, a coautoria de artigos deve ser baseada simultaneamente nos seguintes critérios:

1. Contribuições substanciais para a conceção ou desenho do trabalho, ou aquisição, análise ou interpretação dos dados para o trabalho \&...

2. Escrita do trabalho ou revisão crítica do mesmo \&...

3. Aprovação final da versão para publicação \&...

4. Assumir a responsabilidade por todos os aspetos do trabalho, assegurando que questões relacionadas com a sua validade ou integridade são apropriadamente investigadas e resolvidas.

Estes critérios visam esclarecer as funções e responsabilidades dos autores e colaboradores. Segundo a ICMJE, todos os 4 critérios devem ser cumpridos em simultâneo e o imcumprimento de qualquer um desses critérios, segunifica que não devem ser considerados como autores, mas sim como colaboradores e como tal listados na secção dos agradecimentos. Por exemplo não constituem critérios per se para autoria: i) ser diretor de serviço ou de departamento; ii) apoio administrativo ou financeiro (e.g., recolha de fundos monetários e de dados); iii) apoio linguístico, técnico ou de escrita (e.g., edição de imagens ou gramatical do idioma); iv) direção administrativa de um grupo de investigação; e v) dar um conselho científico, fornecer espaço ou reagentes.

Merece particular clarificação o ponto 1. As diretrizes para Good Publication Practice for Communicating CompanySponsored Medical Research (GPP3) (2015a) definem como uma contribuição substancial para uma publicação ou apresentação "aquela que é intelectualmente importante, ao invés de uma simples assistência técnica, sem a qual o trabalho, ou uma parte importante do trabalho, não poderia ter sido concluído ou o manuscrito não poderia ter sido escrito e submetido para publicação". Naturalmente esta definição não está isenta de limites de difícil aplicação ou compreensão. Tanto os autores, como os colaboradores têm a responsabilidade de criar uma publicação biomédica que seja precisa e fiel aos resultados do estudo, mas apenas os autores devem fornecer uma contribuição substancial para o trabalho e são responsáveis por essa contribuição (Stocks et al., 2018).

Muitas revistas científicas adotaram essas diretrizes, e outros grupos profissionais, como a International Society for Medical Publication Professionals (ISMPP), a World Association of Medical Editors (WAME) e a International Federation of Pharmaceutical Manufacturers e outras Associações citam estas diretrizes nas suas próprias recomendações; por exemplo mais de 1000 revistas estão descritas como respeitantes destas guidelines (Battisti et al., 2015; Hamilton and Royer, 2003; Jacobs and Wager, 2005; Marušić et al., 2011; World Association of Medical Editors WAME, 2021). 
Também de acordo com a WAME, a autoria implica uma contribuição intelectual significativa para o trabalho, algum papel na redação e na revisão final do documento, mas os papéis da autoria podem variar. Refere ainda que quem "será o autor e em que sequência ficará, tal deve ser determinado pelos participantes no início do processo de investigação, para evitar disputas e mal-entendidos que podem atrasar ou impedir a publicação de um artigo". O Committee on Publication Ethics (COPE) exalta também que devem estar em vigor "políticas claras (que permitem a transparência sobre quem contribuiu para o trabalho e em que capacidade) para os requisitos de autoria e contribuição (i.e., authorship e contributorship), bem como processos para gestão de disputas potenciais.

Em algumas áreas temos observado verdadeiros registos épicos em termos do número de autores. Acredita-se que se tenham dado os primeiros passos na área da física de partículas mas a hiperautoria (i.e., hyperauthorship) está a difundir-se para outras áreas científicas como epidemiologia e alterações climáticas (Singh Chawla, 2019). Por exemplo um registo épico aconteceu num artigo de 33 páginas das quais 9 páginas (incluindo referências) são de texto e 24 páginas são de 5154 autores e respetivas afiliações (Khachatryan et al., 2015).

O Comité de Ética para a Publicação da WAME (World Association of Medical Editors WAME, 2021) sugere que "as revistas científicas devem publicar orientações sobre o que constitui autoria", e nesse pressuposto damos o primeiro passo com este editorial que explora e explicita toda a política da RevSALUS sobre esta matéria. Reajustaremos as nossas instruções para os autores da RevSALUS de modo que vigore em cada artigo e de forma clara a contribuição de cada autor e deste modo promovermos a transparência das coautorias.

Num próximo editorial discutiremos sobre a ordem a atribuir aos autores e sobre os abusos a evitar nas coautorias, como são os casos do ghost authorship, gift authorship e o guest authorship.

\section{CONFLITO DE INTERESSES E FINANCIAMENTO}

O autor declara não ter afiliações ou envolvimento financeiro que seja gerador de conflito de interesses.

\section{REFERÊNCIAS BIBLIOGRÁFICAS:}

Good Publication Practice for Communicating Company-Sponsored Medical Research: GPP3. Annals of internal medicine 163:461-464, 2015a.

Recomendações para elaboração, redação, edição e publicação de trabalhos acadêmicos em periódicos médicos. Epidemiologia $e$ Serviços de Saúde 24:577-601, 2015b.

Battisti WP, Wager E, Baltzer L, Bridges D, Cairns A, Carswell Cl, Citrome L, Gurr JA, Mooney LA, Moore BJ, Peña T, Sanes-Miller CH, Veitch $\mathrm{K}$, Woolley $\mathrm{KL}$ and Yarker YE. Good Publication Practice for Communicating Company-Sponsored Medical Research: GPP3. Annals of internal medicine 163:461-464, 2015.

Hamilton CW and Royer M. AMWA position statement on the contributions of medical writers to scientific publications. AMWA J 18:13-16, 2003.

International Committee of Medical Journal Editors (ICMJE). Recommendations for the conduct, reporting, editing, and publication of scholarly work in medical journals, Disponível em http://www.icmje.org/icmje-recommendations.pdf, 2019.

Jacobs A and Wager E. European Medical Writers Association (EMWA) guidelines on the role of medical writers in developing peerreviewed publications. Current medical research and opinion 21:317-321, 2005.

Khachatryan V, Sirunyan AM, Tumasyan A,.... and Collaboration CMS. Observation of the rare Bs0 $\nabla \mu+\mu$ - decay from the combined analysis of CMS and LHCb data. Nature 522:68-72, 2015.

Marušić $A$, Bošnjak $L$ and Jerončić $A$. A systematic review of research on the meaning, ethics and practices of authorship across scholarly disciplines. PloS one 6:e23477, 2011.

Singh Chawla D. Hyperauthorship: global projects spark surge in thousand-author papers. Nature, 2019

Stocks A, Simcoe D, Toroser D and DeTora L. Substantial contribution and accountability: best authorship practices for medical writers in biomedical publications. Current medical research and opinion 34:1163-1168, 2018.

World Association of Medical Editors (WAME). Recommendations on publication ethics policies for medical journals. Disponível em: https://wame.org/recommendations-on-publication-ethics-policiesfor-medical-journals, WAME - World Association of Medical Editors, 2021. 